\title{
Electronic Device Screen Time and Meibomian Gland Morphology in Children
}

\author{
Özkan Kocamiş, MD; Emine Temel, MD; Nazife Aşikgarip, MD; Kemal Örnek, MD
}

Department of Opthalmology, Kırşehir Ahi Evran University School of Medicine, Kırşehir, Turkey

ORCID:

Özkan Kocamış: https://orcid.org/0000-0003-0353-457X

Emine Temel: https://orcid.org/0000-0001-6302-9175

\section{Abstract}

Purpose: To investigate changes in meibomian gland morphology and impact of electronic device usage time on meibomian glands in pediatric age group.

Methods: In this prospective study, 149 eyes of 149 children were enrolled. The participants also completed the Standard Patient Evaluation of Eye Dryness (SPEED) questionnaire and provided information regarding weekly hours spent in front of a digital screen. Meibography was performed in all subjects. Grading of images was evaluated using a previously validated 5-point meiboscale $(0-4)$ for meibomian gland atrophy and a 3-point scale for meibomian gland tortuosity $(0-2)$.

Results: Of the 149 enrolled children, 83 (55.7\%) were female and 66 (44.3\%) male. The mean age was $13.0 \pm 3.0$ (range, $5-18$ ) years. The mean loss of meibomian gland area was $20.80 \pm 9.32 \%$. The mean meiboscore was $1.20 \pm 0.58$ for gland atrophy and the mean tortuosity score was $0.99 \pm 0.62$. The mean screen time was $29.32 \pm 16.18 \mathrm{hr} /$ week . There was a weak and significantly positive correlation between loss of meibomian gland area and screen time $(r=0.210, P=0.010)$. There was a weak and significantly positive correlation between meiboscore for gland atrophy and screen time $(r=0.188, P=0.022)$. We found a weak but significantly positive correlation between meibomian gland tortuosity and screen time $(r=0.142, P=0.033)$.

Conclusion: Meibomian gland morphology may show changes in pediatric age group and excessive screen time may be a factor triggering these changes in gland morphology.

Keywords: Meibography; Meibomian Gland; Pediatric Age; SPEED Score

J Ophthalmic Vis Res 2021; 16 (4): 531-537

\section{INTRODUCTION}

Meibomian gland secretion plays a crucial role in maintaining ocular surface health. The lipid

Correspondence to:

Emine Temel, MD. Department of Ophthalmology, Kırșehir Ahi Evran Training and Research Hospital, Kırșehir 40100, Turkey.

E-mail: emine912@hotmail.com

Received: 30-11-2020 Accepted: 03-09-2020

\section{Access this article online}

Website: https://knepublishing.com/index.php/JOVR

DOI: 10.18502/jovr.v16i4.9741 secretion of the meibomian glands is the main component of the outer layer of tear film and plays an important role in tear film stability. ${ }^{[1]}$ Meibomian gland dysfunction (MGD) is a chronic, diffuse abnormality of the meibomian glands, commonly characterized by duct obstruction and/or changes in the glandular secretion. Due to the inflammatory

This is an open access journal, and articles are distributed under the terms of the Creative Commons Attribution-NonCommercial-ShareAlike 4.0 License, which allows others to remix, tweak, and build upon the work non-commercially, as long as appropriate credit is given and the new creations are licensed under the identical terms. 
and obstructive nature of chronic MGD, meibomian gland atrophy may develop over time. ${ }^{[2]}$ Meibomian gland atrophy can be associated with a systemic disease or can be part of the normal aging process. It has been previously reported that the incidence of meibomian gland atrophy increases with age. ${ }^{[1,3,4]}$ However, little is known about the prevalence of meibomian gland atrophy in pediatric age group and there are few reports in the literature demonstrating alterations of meibomian glands in this population. ${ }^{[5-7]}$

The use of electronic devices (smartphones, tablets, and computers) has increased rapidly during the past decade. The long-term ocular effects of these devices are unknown. However, a range of short-term ocular surface discomfort as well as visual discomfort have been reported. ${ }^{[8-10]}$ Ocular surface discomfort includes dryness, stinging, burning, itchiness, and irritation of the eye. Visual discomfort includes blurred vision, difficulty refocusing when shifting focus from one distance to another, headache, and eye strain. Moon et al reported that smartphone use in children was strongly associated with pediatric dry eye disease. ${ }^{[11]}$

First described by Arita et al, noncontact infrared meibography is a relatively new technique used to obtain information about the morphologic characteristics of the meibomian glands. ${ }^{[1,6,12]}$ It is a noninvasive, rapid, and patient-friendly examination method that visualizes the silhouette of meibomian glands through infrared illumination of the everted eyelid from the conjunctival side. ${ }^{[1,12]}$

In this study, we aimed to investigate the meibomian gland morphology in pediatric age group and the effect of electronic device usage time on meibomian glands.

\section{METHODS}

In this prospective study, 149 eyes of 149 patients who presented to the University Hospital Outpatient Clinic for routine eye examination were included. Informed consent was obtained from parents of all participants in accordance with the Institutional Review Board Committee's approval and adherence to the declaration of Helsinki.

The participants under the age of 18 who could cooperate with image acquisition of meibomian glands were included. Exclusion criteria were history of dry eye disease, presence of MGD, presence of allergic conjunctivitis, contact lens use, past ocular surgery, ongoing medication use, and presence of any ocular or systemic disease.

Biomicroscopic slit-lamp examination was performed in all subjects. The participants also completed the Standard Patient Evaluation of Eye Dryness (SPEED) questionnaire and provided information regarding the number of hours per week spent in front of a digital screen.

The upper eyelid was everted and noncontact meibography was performed in all subjects using corneal topographic device (Sirius, CSO, Florence, Italy) with Phoenix-Meibography Imaging software module. Only right eyes were included in the study. The morphological characteristics of the meibography images were evaluated by blinded physicians.

Previously validated 5-point meiboscale (0-4) for meibomian gland atrophy was used to grade the images. ${ }^{[13]}$ Meiboscale is as follows: grade 0 : normal meibomian glands, grade 1: $\leq 25 \%$ gland atrophy, grade 2: $26-50 \%$ gland atrophy, grade 3: $51-75 \%$ gland atrophy, and grade 4 : $>75 \%$ gland atrophy [Figure 1]. Grading of meibomian gland tortuosity was also performed using a 3-point scale (0-2) with tortuosity defined as a >45-degree angle of the meibomian gland; grade 0: no distortion, grade 1: 1-4 glands distorted, and grade $2: \geq 5$ glands distorted [Figure 1]. . $^{14]}$

The first noninvasive tear film break-up time (NIFBUT) and average noninvasive tear film break-up time (NIBUT) were also evaluated by the software for each case.

All statistical analyses were performed using IBM SPSS 15.0 (SPSS Inc., Chicago, IL, USA). Testing for normal distribution was performed by Kolmogorov-Smirnov test. The mean and standard deviation values were provided for numerical variables with a normal distribution. For numerical variables with normal distribution, the difference between the two groups was determined by $t$-test in independent groups. The Pearson's correlation coefficient was used to measure the strength of a linear association between two variables, where the value $r=1$ means a perfect positive correlation, and the value $r=-$ 1 means a perfect negative correlation. Statistical significance was defined at a level of $5 \%(P<$ 0.05). 


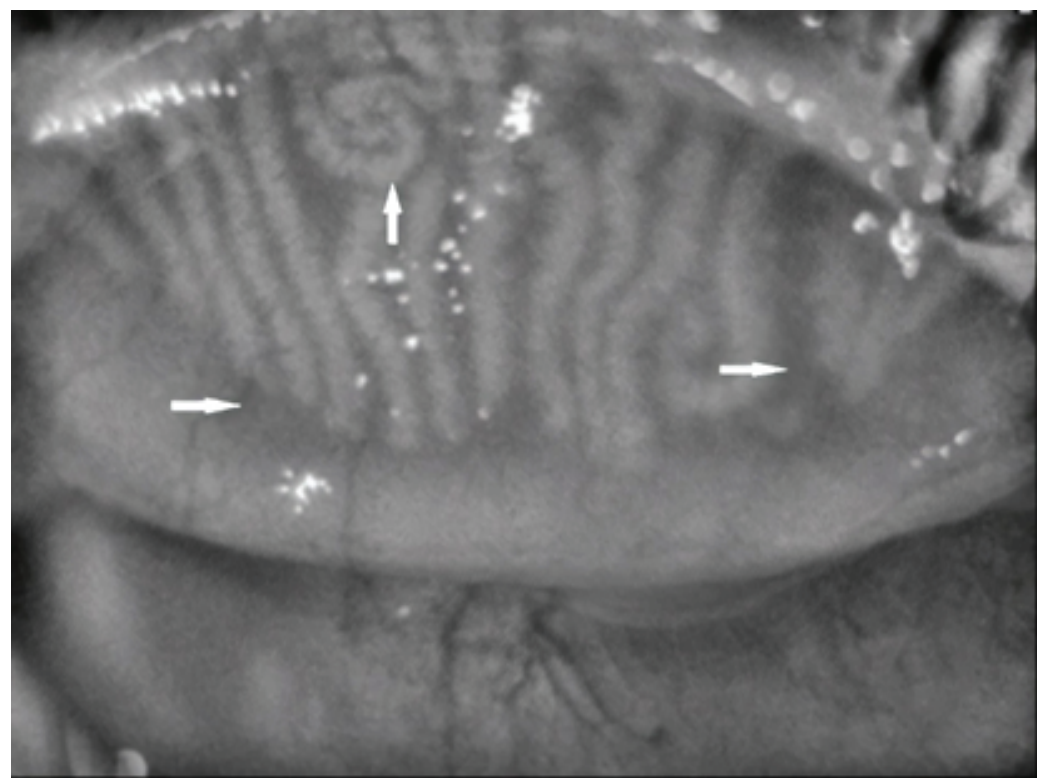

Figure 1. Horizontal arrows show atrophic areas and vertical arrow shows increasing tortuosity.

\section{RESULTS}

Of the 149 children included in the study, 83 $(55.7 \%)$ were female and $66(44.3 \%)$ male. The mean age was $13.0 \pm 3.0$ (range, $5-18$ ) years. The mean loss of meibomian gland area was $20.80 \pm 9.32 \%$. The mean meiboscale was 1.20 \pm 0.58 for gland atrophy and the mean tortuosity score was $0.99 \pm 0.62$. One-hundred thirty-seven (91.4\%) participants had evidence of meibomian gland atrophy (meiboscale $\geq 1$ ) and 120 (80.5\%) had evidence of meibomian gland tortuosity (tortuosity score $\geq 1$ ). Demographics and clinical characteristics of the participants are summarized in Table 1.

\section{SPEED Score}

The average SPEED score was $0.93 \pm 3.55$ with a range from 0 to 20. No significant association was found between the SPEED score and loss of meibomian gland area $(P=0.236)$, meiboscale for gland atrophy $(P=0.218)$, neither for gland tortuosity $(P=0.596)$.

\section{Noninvasive Tears Film Break-up Time}

The mean NIF-BUT was $11.49 \pm 5.42$ s ranging from 2 to $17 \mathrm{~s}$. The average NIBUT was $11.94 \pm 5.04 \mathrm{~s}$ with a range from 2 to $17 \mathrm{~s}$.

\section{Screen Time}

The mean screen time was $29.32 \pm 16.18 \mathrm{hr} /$ week and ranged from 7 to $70 \mathrm{hr}$. There was a weak and significantly positive correlation between the loss of meibomian gland area and screen time $(r=0.210$, $P=0.010)$. There was a weak and significantly positive correlation between meiboscale for gland atrophy and screen time $(r=0.188, P=0.022)$. We found a weak and significantly positive correlation between meibomian gland tortuosity and screen time ( $r=0.142, P=0.033$ ). Correlation graphs of meibomian gland parameters and screen time are shown in Figure 2.

\section{Subgroup Analysis By Gender}

The mean screen time per week was $30.03 \pm$ $15.74 \mathrm{hr}$ in females and $26.68 \pm 13.95 \mathrm{hr}$ in males ( $P=0.177$ ). The mean loss of meibomian gland area was $21.22 \pm 9.15 \%$ in females and $20.26 \pm 9.57 \%$ in males. There was no statistically significant difference between genders in terms of loss of meibomian gland area $(P=0.538)$. The mean meiboscale for gland atrophy was 1.15 \pm 0.63 in males and $1.25 \pm 0.53$ in females. The mean tortuosity score was $0.89 \pm 0.65$ in males and $1.07 \pm 0.57$ in females. No statistically significant difference was found between genders for atrophy and tortuosity $(P=0.294$ and $P=0.081$, respectively). 

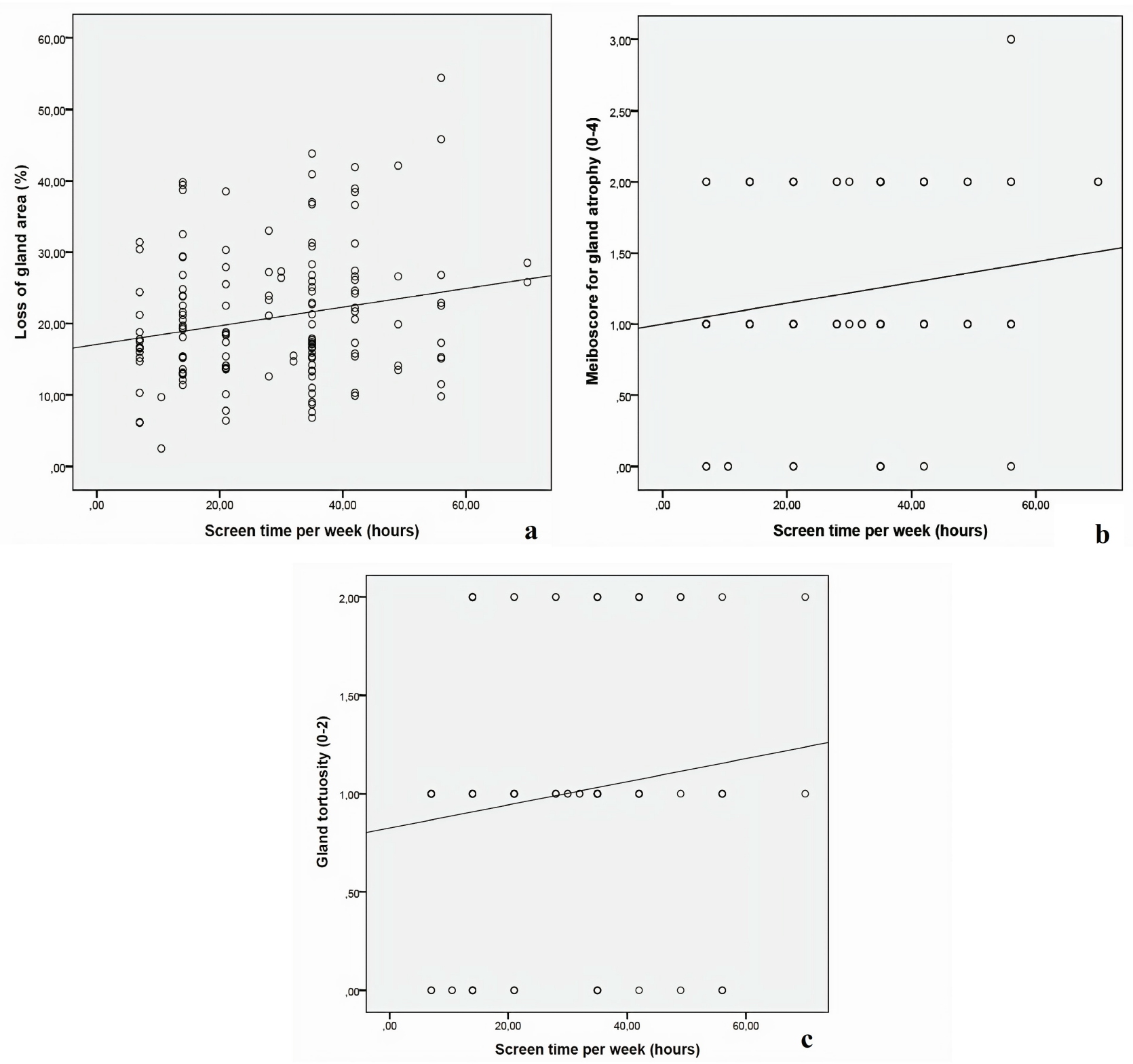

Figure 2. (a) Correlation graph of loss of meibomian gland area and screen time. (b) Correlation graph of meiboscale for gland atrophy and screen time. (c) Correlation graph of meibomian gland tortuosity and screen time.

The average NIBUT was $12.5 \pm 4.81 \mathrm{~s}$ in females and $11.2 \pm 5.25 \mathrm{~s}$ in males $(P=0.112)$. The mean NIFBUT was $11.94 \pm 5.42 \mathrm{~s}$ in females and $10.91 \pm 5.41 \mathrm{~s}$ in males $(P=0.247)$. The distribution of parameters in each gender is presented in Table 2.

\section{DISCUSSION}

In the current study, we assessed alterations of meibomian gland morphology in pediatric population. We also investigated the correlation between electronic device usage time with the parameters measured.
Alterations in meibomian gland morphology are considered to be related to glandular function disorders. Recent studies have shown that gland dropout occurs as an age-related atrophic process. ${ }^{[1,2]}$ The prevalence of MGD is known to be significantly increased in older age groups. In this study, although the enrolled pediatric population had no history of dry eye disease or MGD, approximately $91.4 \%$ showed some evidence of meibomian gland atrophy. This is a higher rate compared to previous studies. Gupta et al evaluated the 
Table 1. Demographics and clinical characteristics for all participants

\section{Sex n (\%)}

Female

Male

Age (yr)

Mean \pm SD

$13.0 \pm 3.0$

(range)

Meiboscore for gland atrophy $\mathbf{n}(\%)$

Grade 0

Grade 1

Grade 2

Grade 3

Grade 4

Tortuosity score $\mathbf{n}(\%)$

Grade 0

Grade 1

Grade 2

SD, standard deviation; yr, year

Table 2. Gender distribution of study parameters

\begin{tabular}{lccc}
\hline Parameter & Females & Males & $P$-value \\
\hline Screen time (hr) per week (Mean \pm SD) & $30.03 \pm 15.74$ & $26.68 \pm 13.95$ & 0.177 \\
Loss of gland area (\%) (Mean \pm SD) & $21.22 \pm 9.15 \%$ & $20.26 \pm 9.57 \%$ & 0.538 \\
Meiboscore for gland atrophy (Mean \pm SD) & $1.25 \pm 0.53$ & $1.15 \pm 0.63$ & 0.294 \\
Tortuosity score (Mean \pm SD) & $1.07 \pm 0.57$ & $1.89 \pm 0.65$ & 0.081 \\
NIF-BUT (s) (Mean \pm SD) & $11.94 \pm 5.42$ & $10.91 \pm 5.41$ & 0.247 \\
Average NIBUT (s) (Mean \pm SD) & $12.5 \pm 4.81$ & $11.2 \pm 5.25$ & 0.112
\end{tabular}

SD, standard deviation; NIF-BUT, noninvasive first tear film break-up time; NIBUT, noninvasive tear film break-up time *Statistically significant $p$-value

prevalence of meibomian gland atrophy and gland tortuosity in a pediatric population. ${ }^{[13]}$ They showed that $42 \%$ of the subjects had evidence of meibomian gland atrophy. ${ }^{[15]}$ This observation suggests that meibomian gland atrophy is a common condition and can be found in asymptomatic pediatric population.

The question at this point is unveiling the causative factors in meibomian gland atrophy in the pediatric population. Increasing numbers of dry eye disease and MGD in the general population suggest that environmental factors may trigger this condition in patients with subclinical MGD. Considering that pediatric cases are less symptomatic than adults, long-term outcomes can be improved with regular follow-ups in pediatric patients at risk.

In a study by Gupta et al, it was shown that $42 \%$ of the subjects had evidence of meibomian gland atrophy and $37 \%$ had evidence of meibomian gland tortuosity. ${ }^{[15]}$ They did not find an increase in tortuosity in $63 \%$ of the cases. In our study, 137 subjects (91.4\%) had some degree of meibomian gland atrophy and 120 (80.5\%) had some degree of meibomian gland tortuosity. We did not find an increase in tortuosity in $19.5 \%$ of the cases. Gupta et al also reported that the mean meiboscale was $0.58 \pm 60.80$ for gland atrophy and $0.45 \pm 0.64$ for tortuosity. ${ }^{[14]}$ According to our results, the mean 
meiboscale was $1.20 \pm 0.58$ for gland atrophy and the mean tortuosity score was $0.99 \pm 0.62$. Wu et al divided their participants into two groups: child (age range, 3 to 11 years) and adolescent (age range, 12 to 18 years) groups. ${ }^{[1]}$ They reported that meibomian gland loss was observed in both groups, but the meiboscale was not significantly different between the two groups. ${ }^{[1]}$

In addition, we found that changes in meibomian gland morphology were not significantly different between males and females. Contrary to our findings, Mizoguchi et al evaluated the morphology and function of meibomian glands in junior high school students at 15 years of age and reported that changes to the meibum quality were significantly greater in males than in females. ${ }^{[14]}$ According to the authors, the differences may be due to sex hormones like androgens and estrogens. Both androgens and estrogens were previously shown to affect meibomian gland function. ${ }^{[16-22]}$

In this study, children with a previous diagnosis of DED were excluded and the SPEED questionnaire was used to screen for symptoms suggestive of MGD and dry eye. Based on the criteria reported by Asiedu et al, ${ }^{[22]}$ the majority of our study population fell into the asymptomatic group. Therefore, the results of our study support that there may be atrophy in the meibomian glands in pediatric population even in asymptomatic subjects. Han et al have shown that children with objective clinical findings of dry eye disease, similar to those seen in adult patients, tend to be less symptomatic compared to adults. ${ }^{[23]}$ This demonstrates the importance of follow-up visits for MGD in children who are at risk of developing ocular surface disease.

Today, the population is increasingly more dependent on their digital devices like computers, tablets, and smartphones. This also affects the pediatric population as they spend excessive time on laptops and tablet devices at school and at home. Long-term visual display terminal work, especially for more than 4 hr daily, was associated with a high incidence of dry eye disease. ${ }^{[25]}$ Fenga et al reported that the development of ocular complaints in visual display terminal workers was correlated with MGD. ${ }^{[25]}$ MGD has been generally considered the main reason for evaporative dry eye. The use of electronic devices that make people keep their eyes widely open has been recognized to decrease the frequency of blinking and accelerate tear evaporation.
Moon et al evaluated the risk factors of dry eye disease in school children associated with video display terminal use. ${ }^{[26]}$ Their results showed that smartphone use is an important dry eye disease risk factor in children. Moon et al and Gupta et al did not find a significant correlation between the amount of time spent in front of a visual display device and meibomian gland atrophy. ${ }^{[13,26]}$ According to our findings, the risk of developing atrophy and tortuosity in meibomian glands is higher in children when screen time is increased.

Our study has some limitations. The first is the inability to completely observe the meibomian glands in the pediatric age group due to poor compliance in children. Everting the upper eyelid made the examination process very difficult. Second, the studied population was from a single tertiary care center, which might have resulted in selection bias (all patients were living in a city with a population of 100,000 ).

In summary, there was a significant, albeit weak, relationship between the time spent in front of screens and the meibomian gland changes in pediatric age group. In today's digital information age, it is recommended for children to be followed and monitored for meibomian gland changes.

\section{Financial Support and Sponsorship}

Nil.

\section{Conflicts of Interest}

There are no conflicts of interest.

\section{REFERENCES}

1. Wu Y, Li H, Tang Y, Yan X. Morphological evaluation of meibomian glands in children and adolescents using noncontact infrared meibography. J Pediatr Ophthalmol Strabismus 2017;54:78-83.

2. Knop E, Knop N, Millar T, Obata H, Sullivan DA. The international workshop on meibomian gland dysfunction: report of the subcommittee on anatomy, physiology, and pathophysiology of the meibomian gland. Invest phthalmol Vis Sci 2011;52:1938-1978.

3. Yeotikar NS, Zhu H, Markoulli M, Nichols KK, Naduvilath T, Papas EB. Functional and morphologic changes of meibomian glands in an asymptomatic adult population. Invest Ophthalmol Vis Sci 2016;57:3996-4007.

4. Den S, Shimizu K, Ikeda T, Tsubota K, Shimmura S, Shimazaki J. Association between meibomian gland changes and aging, sex, or tear function. Cornea 2006;25:651-655. 
5. Arita R, Itoh K, Maeda S, Maeda K, Amano S. A newly developed noninvasive and mobile pen-shaped meibography system. Cornea 2013;32:242-247.

6. Shirakawa R, Arita R, Amano S. Meibomian gland morphology in Japanese infants, children, and adults observed using a mobile penshaped infrared meibography device. Am J Ophthalmol 2013;155:1099_ 1103.e1091.

7. Maducdoc MM, Haider A, Nalbandian A, Youm JH, Morgan PV, Crow RW. Visual consequences of electronic reader use: a pilot study. Int Ophthalmol 2017;37:433-439.

8. Long J, Cheung R, Duong S, Paynter R, Asper L. Viewing distance and eyestrain symptoms with prolonged viewing of smartphones. Clin Exp Optom 2017;100:133-137.

9. Moon JH, Kim KW, Moon NJ. Smartphone use is a risk factor for pediatric dry eye disease according to region and age: a case control study. BMC Ophthalmol 2016;16:188194.

10. Uchino M, Schaumberg DA, Dogru M, Uchino Y, Fukagawa $\mathrm{K}$, Shimmura S, et al. Prevalence of dry eye disease among Japanese visual display terminal users. Ophthalmology 2008;115:1982-1988.

11. Pult $\mathrm{H}$, Riede-Pult B. Comparison of subjective grading and objective assessment in meibography. Cont Lens Anterior Eye 2013;36:22-27.

12. Arita R, Itoh K, Maeda S, Maeda K, Tomidokoro A, Amano $\mathrm{S}$. Association of contact lens-related allergic conjunctivitis with changes in the morphology of meibomian glands. Jpn J Ophthalmol 2012;56:14-19.

13. Gupta PK, Stevens MN, Kashyap N, Priestley Y. Prevalence of meibomian gland atrophy in a pediatric population. Cornea 2018;37:426-430.

14. Mizoguchi T, Arita R, Fukuoka S, Morishige N. Morphology and function of meibomian glands and other tear film parameters in junior high school students. Cornea 2017;36:922-926.

15. Khandelwal P, Liu S, Sullivan DA. Androgen regulation of gene expression in human meibomian gland and conjunctival epithelial cells. Mo/ Vis 2012;18:1055-1067.

16. Sullivan DA, Jensen RV, Suzuki T, Richards SM. Do sex steroids exert sex-specific and/or opposite effects on gene expression in lacrimal and meibomian glands? Mol Vis 2009;15:1553-1572.

17. Schirra F, Richards SM, Liu M, Suzuki T, Yamagami $H$, Sullivan DA. Androgen regulation of lipogenic pathways in the mouse meibomian gland. Exp Eye Res 2006;83:291296.

18. Cermak JM, Krenzer KL, Sullivan RM, Dana RM, Sullivan DA. Is complete androgen insensitivity syndrome associated with alterations in the meibomian gland and ocular surface? Cornea 2003;22:516-521.

19. Sullivan BD, Evans JE, Cermak JM, Krenzer KL, Dana MR, Sullivan DA. Complete androgen insensitivity syndrome: effect on human meibomian gland secretions. Arch Ophthalmol 2002;120:1689-1699.

20. Sullivan DA, Sullivan BD, Evans JE, Schirra F, Yamagami $H$, Liu $M$, et al. Androgen deficiency, meibomian gland dysfunction, and evaporative dry eye. Ann N Y Acad Sci 2002;966:211-222.

21. Sullivan DA, Sullivan BD, Ullman MD, Rocha EM, Krenzer KL, Cermak JM, et al. Androgen influence on the meibomian gland. Invest Ophthalmol Vis Sci 2000;41:3732-3742.

22. Asiedu K, Kyei S, Mensah SN, Ocansey S, Abu LS, Kyere EA. Ocular surface disease index (OSDI) versus the standard patient evaluation of eye dryness (SPEED): a study of a nonclinical sample. Cornea 2016;35:175-180.

23. Han SB, Yang HK, Hyon JY, Hwang JM. Children with dry eye type conditions may report less severe symptoms than adult patients. Graefes Arch Clin Exp Ophthalmol 2013;251:791-796.

24. Kojima T, Ibrahim OM, Wakamatsu T, Tsuyama A, Ogawa $J$, Matsumoto $\mathrm{Y}$, et al. The impact of contact lens wear and visual display terminal work on ocular surface and tear functions in office workers. Am J Ophthalmol 2011;152:933-940.

25. Fenga C, Aragona $P$, Cacciola A, Spinella R, Niola $\mathrm{CD}$, Ferreri $\mathrm{F}$, et al. Meibomian gland dysfunction and ocular discomfort in video display terminal workers. Eye 2008;22:91-95.

26. Moon JH, Lee MY, Moon NJ. Association between video display terminal use and dry eye disease in school children. J Pediatr Ophthalmol Strabismus 2014;51:87-92. 